\title{
Comportamiento de los niveles de troponina I de alta sensibilidad en pacientes con enfermedad renal crónica estadio 5 en hemodiálisis*
}

\section{Behavior of high sensitivity Troponin I levels in patients with chronic kidney disease stage 5 on hemodialysis*}

\author{
Ana María Barón, Ángel García, Marietta Juan Guardela, \\ Martha Patricia Rodríguez, Camilo González, Pilar Alarcón, \\ María Camila Betancur • Bogotá, D.C. (Colombia)
}

\section{Resumen}

Introducción: los pacientes con enfermedad renal crónica (ERC) tienen mayor prevalencia de elevación de troponina, incluso en ausencia de síndrome coronario agudo. El objetivo de este estudio fue evaluar el comportamiento de la troponina I ultrasensible en pacientes con ERC antes y después de una sesión de hemodiálisis.

Diseño: pseudoexperimento.

Metodología: se incluyeron pacientes mayores de 18 años con ERC estadio 5 en hemodiálisis. Pacientes con condición cardiaca activa, uso de dispositivo cardiaco, intervención coronaria percutánea o historia de cirugía cardiaca se excluyeron del estudio. Los valores de troponinas superiores al percentil $99^{\circ}$ fueron considerados positivos antes de la hemodiálisis y una elevación de más del $20 \%$ fue considerada positiva de acuerdo con la definición universal de infarto de miocardio. Todos los pacientes firmaron un consentimiento informado.

Resultados: se incluyeron 33 pacientes con edad promedio de 56 años. Un paciente presentó troponina elevada antes (3\%) y dos pacientes la elevaron después de la diálisis $(6 \%)$. No hubo diferencias estadísticas entre ambos grupos (p-valor 0.8506). El mismo paciente que aumentó la troponina antes la elevó posdiálisis, lo que significa que sólo un paciente elevó troponina durante la sesión de hemodiálisis.

Conclusión: se encontró una baja incidencia de troponina I anormal en pacientes con ERC en hemodiálisis con una diferencia no significativa de elevación asociada a la diálisis. No hubo relación con comorbilidades. Estos resultados demuestran la importancia de interpretar las elevaciones de troponina como una condición de riesgo sin considerar su elevación como consecuencia de su enfermedad renal. (Acta Med Colomb 2017; 42: 210-214).

Palabras clave: troponina, hemodiálisis, enfermedad renal crónica, síndrome coronario agudo, prevalencia.

\section{Abstract}

Introduction: patients with chronic kidney disease (CKD) have a higher prevalence of troponin elevation, even in the absence of acute coronary syndrome. The objective of this study was to evaluate the behavior of ultrasensitive troponin I in patients with CKD before and after a hemodialysis session.

Design: pseudoexperiment.

Methodology: patients older than 18 years with stage 5 CKD in hemodialysis were included. Patients with active heart condition, cardiac device use, percutaneous coronary intervention or history of cardiac surgery were excluded from the study. Troponin values above the 99th percentile were considered positive before hemodialysis and an elevation of more than $20 \%$ was considered positive according to the universal definition of myocardial infarction. All patients signed an informed consent.

Results: 33 patients with an average age of 56 years were included. One patient presented high
*Trabajo ganador del Premio "Trabajo de Investigación Clínica - Internistas", otorgado por el X Congreso SOLAMI - XXVI Congreso ACMI-ACP, Cartagena $17-20$ de agosto de 2017.

Dra. Ana María Barón: Internista-Cardióloga; Dr. Ángel García: MsC. Internista-Cardiólogo. Magister en Epidemiología Clínica; Dra. Marietta Juan Guardela: Internista-Fellow Cardiología; Dra. Martha Patricia Rodríguez: Internista-Nefróloga; Dr. Camilo González: Internista-Nefrólogo; Dra. Pilar Alarcón: Internista; Dra. María Camila Betancur: Médico General. Departamento de Medicina Interna, Hospital Universitario San Ignacio, Pontificia Universidad Javeriana. Bogotá, D.C. (Colombia).

Correspondencia: Dra. Pilar Alarcón. Bogotá, D.C. (Colombia)

E-mail: alarconroblesp@gmail.com

Recibido: 30/VI/2017 Aceptado: 19/VIII/2017 
troponin before (3\%) and two patients had it elevated after dialysis (6\%). There were no statistical differences between the two groups (p-value 0.8506). The same patient with increased troponin before, elevated it after dialysis, which means that only one patient raised troponin during the hemodialysis session.

Conclusion: a low incidence of abnormal troponin I was found in patients with CKD on hemodialysis with a non-significant difference in elevation associated with dialysis. There was no relationship with comorbidities. These results demonstrate the importance of interpreting troponin elevations as a risk condition without considering its elevation as a consequence of kidney disease. (Acta Med Colomb 2017; 42: 210-214).

Key words: troponin, hemodialysis, chronic kidney disease, acute coronary syndrome, prevalence.

\section{Introducción}

La enfermedad cardiovascular representa aproximadamente $50 \%$ de las causas de muerte en los pacientes con enfermedad renal crónica (ERC) en diálisis, constituyendo un factor de riesgo en el desarrollo de aterosclerosis y eventos cardiovasculares (1). El uso de los marcadores bioquímicos de lesión miocárdica en pacientes con ERC y fuera de escenarios de síndrome coronario agudo, en la actualidad está limitado a su evaluación como factor pronóstico para el desarrollo en el futuro de eventos cardiovasculares; sin embargo, la interpretación sobre la positividad, variación de los niveles, cambios relacionados con la forma de toma y procesamiento de la muestra, y los cambios asociados al tipo de membrana o filtro utilizado durante la sesión de diálisis, son muy controversiales y continúan siendo materia de estudio.

El avance tecnológico de las técnicas para la medición de la troponina I en sangre han mejorado de manera significativa las características operativas de la prueba; sin embargo, este desarrollo ha ocasionado dificultades para la interpretación, análisis y toma de decisiones derivadas de dichos resultados.

En el caso de los pacientes con ERC en hemodiálisis, se ha descrito que la elevación de la troponina en ausencia de evento coronario agudo es un factor de riesgo para muerte cardiovascular convirtiéndose en un marcador pronóstico (2). En nuestro medio es de gran importancia conocer cómo es el comportamiento de la troponina en estos pacientes, para así poder mejorar el proceso diagnóstico en los pacientes en urgencias y posiblemente usarlo como marcador pronóstico en el escenario ambulatorio. El principal objetivo de este estudio es evaluar y describir el comportamiento de los niveles de troponina I de alta sensibilidad en pacientes con ERC en hemodiálisis, adicionalmente se pretende establecer la frecuencia de anormalidad de los valores de troponina I de alta sensibilidad en pacientes con ERC en hemodiálisis, cuantificar los niveles de troponina I de alta sensibilidad en pacientes con ERC en hemodiálisis, determinar la variación de los niveles de troponina I de alta sensibilidad en pacientes estables con ERC en hemodiálisis, antes y después de una sesión de la misma y evaluar la potencial asociación de comorbilidades a los niveles anormales de troponina I de alta sensibilidad.

\section{Material y métodos}

Tipo de diseño: pseudoexperimento. (Estudio de antes y después).

Población de referencia: pacientes con ERC en terapia de reemplazo renal con hemodiálisis.

Criterios de inclusión: pacientes mayores de 18 años con diagnóstico de ERC en hemodiálisis.

\section{Criterios de exclusión}

- Hospitalización por cualquier causa en el último mes.

- Proceso infeccioso de cualquier etiología activo. (Definido como la necesidad de intervención médica, consulta, hospitalización o medicación).

- Presencia de angina o dolor torácico en los últimos 14 días.

- Fibrilación auricular o flutter auricular documentado.

- Uso de marcapasos, cardiodesfibrilador implantable o cardiorresincronizador.

- Cirugía cardiaca de cualquier tipo.

- Haber sido sometido a cateterismo cardiaco o intervención percutánea (angioplastia o colocación de stent).

- Hipertensión arterial no controlada (mayor de 180/100 mmHg), al momento de la evaluación o durante sesión de hemodiálisis por analizar.

- Historia de enfermedad cerebrovascular de cualquier tipo: (accidente cerebrovascular, accidente isquémico transitorio, eventos hemorrágicos cerebrales de cualquier tipo).

- Historia de neoplasia maligna.

- Uso de anticoagulación crónica.

- Disfunción de fístula arteriovenosa y/o catéter de hemodiálisis en el último mes.

- Requerimiento de terapia transfusional (cualquier componente sanguíneo), en el último mes.

- Historia de quemaduras, choques eléctricos, electrocución en el último mes.

- Terapia de radiofrecuencia o crioablación dentro de los últimos 14 días.

- Rabdomiólisis en los últimos tres meses. 
- Miocarditis en los últimos 14 días.

- Tiempo de inicio de hemodiálisis menor a tres meses.

- Embolia pulmonar.

- Inestabilidad hemodinámica o clínica durante la sesión de hemodiálisis (incluye arritmias, dolor torácico, sangrado, necesidad de suspender sesión por cualquier causa, hipotensión medida por TAS menor de $90 \mathrm{mmHg}$ TAD menor $60 \mathrm{mmHg}$, o cualquier condición que amerite intervención médica durante la sesión de hemodiálisis).

- Coagulación del circuito durante la sesión de hemodiálisis.

- Cualquier condición médica que a criterio de los investigadores pudiese eventualmente generar cambios en los niveles de troponina.

\section{Tamaño de muestra}

Se estimó un tamaño de muestra para análisis de diferencia de medias para muestras pareadas, con un error alfa de 0.05 , error beta de 0.2 , poder de $80 \%$, nivel de confianza de $95 \%$, una precisión de 0.0058 , equivalente a $20 \%$ del valor límite superior para considerarse valores normales según la técnica de laboratorio que se usará en el presente estudio con una varianza de 0.029 , estimándose un tamaño de muestra de 33 pacientes, adicionando $15 \%$ por posibles pérdidas para un valor de 39 parejas (39 pacientes, 78 mediciones).

\section{Procedimiento}

La búsqueda de pacientes se realizó en la unidad de nefrología del Hospital Universitario de San Ignacio con el fin de facilitar su inclusión en el estudio. Una vez los sujetos aceptaran participar en el estudio se procedió a la firma del consentimiento informado. Se diligenció el formato de recolección de información descrito en el protocolo del estudio con información sobre variables de interés e identificación de los sujetos. Al ingreso del paciente a sala de hemodiálisis y previo al inicio de la sesión de hemodiálisis, se realizó toma de peso con la báscula calibrada marca SECA modelo 676, sin zapatos, posterior canulación de fístula AV o conexión del sistema a catéter de hemodiálisis y posterior a realizar el proceso de purga con SSN, se tomaron las muestras de sangre bajo las medidas de bioseguridad estipuladas por protocolo de la unidad de diálisis del HUSI, se etiquetó el tubo estéril de la muestra y se enumeró. Las muestras fueron trasladadas de forma inmediata por el personal de laboratorio autorizado o por el investigador para su procesamiento. El mismo procedimiento se repitió al finalizar la sesión de hemodiálisis. Los signos vitales durante la sesión de hemodiálisis (tensión arterial y frecuencia cardiaca) fueron tomados por mecanismo digital incorporado en la máquina de hemodiálisis marca NIKKISO modelo DBB-06. El test usado de troponina fue PATHFAST versión PF1011-K, de Mitsubishi Chemical Medience Corporation.

El proyecto fue presentado al comité de ética e investigaciones de la institución, siendo aprobado para su realización.

\section{Resultados}

Entre marzo de 2014 y septiembre de 2016 se completó el tamaño de muestra previamente estimado de 33 pacientes (Figura 1), en total fueron reclutados 78 pacientes de los cuales 45 pacientes fueron excluidos por los criterios establecidos en el estudio.

El $45.5 \%$ (15) eran hombres y 55.5\% mujeres (18), con edad promedio de 56.2 años en los hombres (DS 18.7, rango de 20-80) y 56.0 en las mujeres (DS 14.5, rango de 28-77). Se encontró que el tiempo promedio de hemodiálisis era de 80 meses, con un peso prediálisis de $65.6 \mathrm{~kg}$ y posdiálisis de $49.4 \mathrm{~kg}$. En cuanto a los antecedentes de la población estudiada un $62.5 \%$ tiene hipertensión arterial, $30 \%$ dislipidemia, $30 \%$ diabetes mellitus, y $77.5 \%$ anemia. Se observó un riesgo cardiovascular estimado mediante Framingham Score ajustado para Colombia de 9.84 en hombres (DS 7.07) y de 8.21 en mujeres (DS 11.28). Las características generales de la población se encuentran descritas en la Tabla 1.

Posterior al análisis descriptivo, se realizaron pruebas de normalidad para los valores de troponina, encontrando mediante el test de Shapiro-Wilk $\mathrm{p}<0.001$ por lo que se decidió utilizar estadística no paramétrica y proceder a comparar los promedios mediante el test de Wilcoxon para muestras pareadas (Tabla 2).

Se puede observar que el promedio de troponina prediálisis es de $0.0064 \mathrm{ng} / \mathrm{dL}$ con una desviación estándar de 0.0056 y posdiálisis de $0.0067 \mathrm{ng} / \mathrm{dL}$ con una desviación estándar de 0.0073 (Figura 2). De las 33 muestras tomadas, el $3 \%$ (un paciente) tuvo troponina prediálisis positiva y $6 \%$ (dos pacientes) troponina postdiálisis positiva. El mismo paciente que presentó elevación de troponina prediálisis, la elevó posdiálisis, lo que significa que sólo un paciente elevó la troponina durante la sesión de hemodiálisis.

No se obtuvo evidencia de diferencia estadísticamente significativa entre los valores encontrados $(p=0.8506)$.

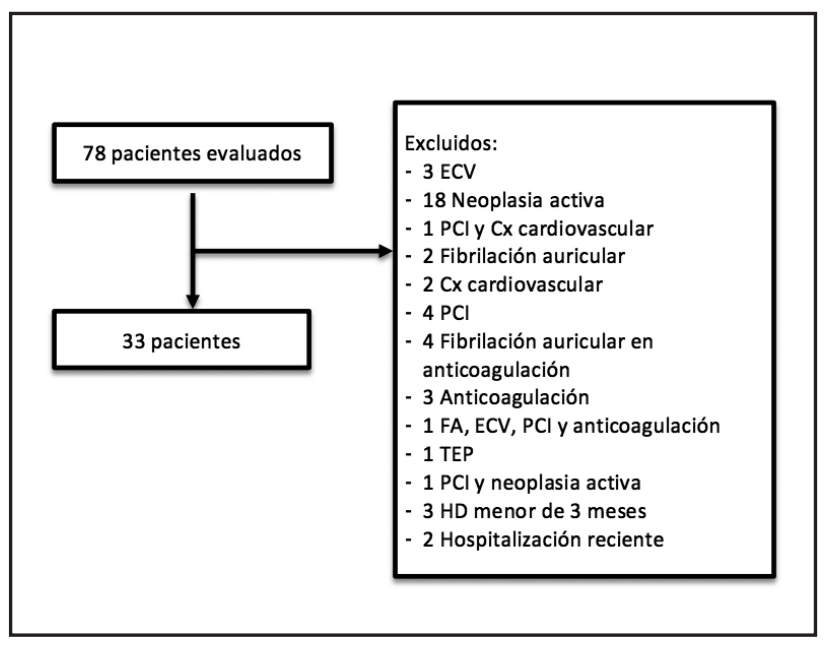

Figura 1. Obtención de la muestra.

ECV: Enfermedad cerebrovascular, PCI: intervención coronaria percutánea, FA: fibrilación auricular, Cx: Cirugía, TEP: tromboembolismo pulmonar, HD: hemodiálisis. 


\section{Discusión}

Conforme a lo encontrado en estudios previos fue mayor el porcentaje de pacientes con ERC en hemodiálisis asintomáticos con troponina negativa (3), sin embargo, existe documentación previa por el grupo de Khumar y colegas de un porcentaje significativo de pacientes con troponina I ultrasensible positiva. En los servicios de urgencias, es frecuente la interpretación de los niveles de troponina I

Tabla 1. Características de la población.

\begin{tabular}{|l|c|c|}
\hline \multicolumn{1}{|c|}{ Variable } & Hombres (15) & Mujeres (18) \\
\hline Edad & 56.2. DS 18.7 & 56.0. DS 14.5 \\
\hline $\begin{array}{l}\text { Tiempo de hemodiálisis } \\
\text { (meses) }\end{array}$ & 59.3. DS 39.3 & 42.72. DS 39.0 \\
\hline Peso prediálisis & 63.7. DS 18.7 & 67.6. DS 12.8 \\
\hline Peso posdiálisis & 62.4. DS 18.6 & 66.4. DS 12.6 \\
\hline Hipertensión arterial & $53 \%$ & $72 \%$ \\
\hline Dislipidemia & $33 \%$ & $27 \%$ \\
\hline Diabetes & $33 \%$ & $55 \%$ \\
\hline Anemia & $100 \%$ & 8.21. DS 11.28 \\
\hline $\begin{array}{l}\text { Framingham Score ajustado } \\
\text { para Colombia }\end{array}$ & 9.84. DS 7.07 & 1.72 \\
\hline Ktv & 1.61 & \\
\hline DS: Desviación estándar. & & \\
\hline
\end{tabular}

Tabla 2. Resultados de troponina.

\begin{tabular}{|l|c|c|}
\hline & \multicolumn{1}{|c|}{$\begin{array}{c}\text { Troponina } \\
\text { predialisis }\end{array}$} & $\begin{array}{c}\text { Troponina } \\
\text { postdialisis }\end{array}$ \\
\hline Promedio - DS & 0.0064. DS 0.0056 & 0.0067. DS 0.0073 \\
\hline Positivas (número) & 1 & 2 \\
\hline Negativas (número) & 32 & p 0.8506 \\
\hline $\begin{array}{l}\text { Test de Wilcoxon muestras } \\
\text { pareadas, hipótesis de dos } \\
\text { colas. }\end{array}$ & \multicolumn{2}{|}{} \\
\hline DS: Desviación estándar & \multicolumn{2}{|}{} \\
\hline
\end{tabular}

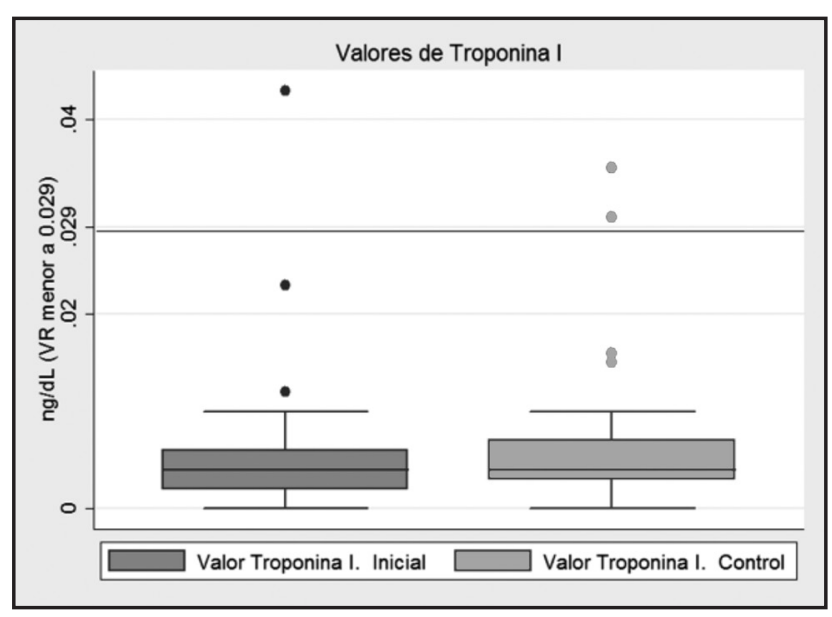

Figura 2. Distribución de los valores de troponina. VR: valor de referencia. elevados en el paciente con ERC en hemodiálisis como un falso positivo, restándole el valor diagnóstico que merece en el contexto de sospecha de síndrome coronario agudo y así mismo en su eventual valor pronóstico, teniendo en cuenta que su elevación confiere aumento de dos a cuatro veces en el riesgo de mortalidad por cualquier causa, mortalidad por causa cardiovascular y riesgo de presentar eventos adversos mayores cardiovasculares (MACE, infarto al miocardio, mortalidad cardiovascular y/o revascularización miocárdica) (4). Sin embargo, en nuestro estudio encontramos, en el grupo de 33 pacientes con ERC en hemodiálisis, que los niveles de troponina I ultrasensible en la mayoría de ellos resultaron negativos, incluso en pacientes con comorbilidad (siendo la HTA, la diabetes y la dislipidemia los más frecuentes) y con riesgo cardiovascular por Framingham ajustado variable con promedio de 9.4 en hombres y 8.21 en mujeres. Los valores posdiálisis demostraron, al mantenerse negativos comparativamente con los niveles prediálisis, que no existe relación entre la realización de la terapia y la positividad o negatividad de la prueba. Tampoco se demostró relación con los niveles de hemoglobina. Si bien se requieren estudios futuros para determinar la asociación entre los niveles elevados de troponina y desenlaces adversos, consideramos que con el uso de un test ultrasensible como en el caso de este trabajo, la positividad de la prueba debe considerarse como una alerta tanto en el contexto agudo como en el seguimiento del paciente crónico, no solo por la relación comprobada de su elevación y desenlaces adversos, sino por la posibilidad que existe de asociación con enfermedad coronaria, isquemia silente o sobrecarga de volumen, y para la cual se requieren estudios posteriores. Igualmente, los resultados abren la puerta a la posibilidad de identificar pacientes en riesgo tras la utilización de la prueba como parte del análisis de riesgo cardiovascular y por qué no, de la implementación de intervenciones sobre el paciente con troponina positiva. Por lo pronto y ante el uso cada vez más frecuente de pruebas ultrasensibles de troponina, debe su positividad generar una alarma en el servicio de urgencias en el paciente con ERC. Es llamativo que en nuestra población, el número de pacientes con troponina I ultrasensible anormal fue bajo y no se asoció a eventos adversos o complicaciones relacionadas con la hemodiálisis durante el periodo del estudio. Estos hallazgos difieren de lo encontrado en otras series posiblemente debido a la exclusión de condiciones preexistentes que se asocian con lesión miocárdica por diversos mecanismos, situación que deberá tenerse en cuenta al momento de la interpretación de los datos en escenarios clínicos diferentes.

\section{Implicación clínica}

Los niveles de troponina I ultrasensible en los pacientes con ERC en hemodiálisis, deben interpretarse de manera cuidadosa en el servicio de urgencias, teniendo en cuenta que en la mayoría de este tipo de pacientes el resultado es negativo, por lo tanto su positividad debe ser valorada suficientemente en el contexto agudo y en la sospecha de 
síndrome coronario agudo con el ánimo de no retrasar intervenciones que puedan favorecer el pronóstico vital de esta población. Se requieren estudios posteriores para considerar la troponina I ultrasensible como marcador pronóstico del paciente con ERC, teniendo en cuenta que su elevación se relaciona con desenlaces desfavorables incluso en el paciente asintomático.

\section{Conclusiones}

El valor de la troponina I en pacientes sometidos a hemodiálisis clínicamente estables y sin condiciones cardiacas activas, en promedio son normales y no presentan diferencias estadísticamente significativas posterior a la sesión de hemodiálisis y son independientes de los valores de hemoglobina o factores de riesgo asociados.

\section{Agradecimientos}

A los miembros de Departamento de Investigación y Desarrollo de Laboratorio Clínico del Hospital Universitario San Ignacio.

A los miembros de Medicina y Enfermería de la Unidad Renal del Hospital Universitario San Ignacio.

\section{Conflicto de intereses}

Loa autores declaran no tener ningún conflicto de intereses.

\section{Referencias}

1. Herzog CA, Ma JZ, Collins AJ. Poor long-term survival after acute myocardial infarction among patients on long-term dialysis. N Engl J Med. 1998; 339: 799.

2. Satyan S, Light RP, Agarwa R. Relationships of N-terminal pro-B-natriuretic peptide and cardiac troponin $\mathrm{T}$ to left ventricular mass and function and mortality in asymptomatic hemodialysis patients. Am J Kidney Dis. 2007; 50: 1009.

3. Wang AY-M, Lai K-N. Use of cardiac biomarkers in end-stage renal disease. $J$ Am Soc Nephrol. 2008; 19: 1643-1652.

4. Michos ED, Wilson LM, Yeh H-C, et al. Prognostic Value of Cardiac Troponin in Patients With Chronic Kidney Disease Without Suspected Acute Coronary Syndrome: A Systematic Review. Ann Intern Med. 2014; 161(7): 1-12. doi:10.7326/ M14-0743.

5. Parmacek MS, Solaro RJ. Biology of the troponin complex in cardiac myocytes. Prog Cardiovasc Dis 2004; 47:159.
6. Katus HA, Remppis A, Looser S, Hallermeier K, Scheffold T, Kubler W. Enzyme linked immunoassay of cardiac troponin $\mathrm{T}$ for the detection of acute myocardial infarction in patients. J Mol Cell Cardiol 1989; 21: 1349.

7. Hamm CW, Ravkilde J, Gerhardt W, Jorgensen P, Peheim E, Ljungdahl L, et al. The prognostic value of serum troponin T in unstable angina. N Engl J Med 1992; 327: 146

8. Jaffe AS. Troponin--past, present, and future. Curr Probl Cardiol. 2012; 37(6): 209-28.

9. Wu A, Christenson RH. Analytical and assay issues for use of cardiac troponin testing for risk stratification in primary care. Clin Biochem 2013; 46: 969.

10. Ricchiuti V, Apple FS. RNA expression of cardiac troponin T isoforms in diseased human skeletal muscle. Clin Chem 1999; 45: 2129-35.

11. Katus HA, Looser S, Hallermayer K, et al. Development and in vitro characterization of a new immunoassay of cardiac troponin T. Clin Chem 1992; 38: 386-93.

12. Omland T, de Lemos JA, Sabatine MS, et al. A sensitive cardiac troponin T assay in stable coronary artery disease. N Engl J Med 2009; 361: 2538-47.

13. Krahn J, Parry DM, Leroux M, et al. High percentage of false positive cardiac troponin I results in patients with rheumatoid factor. Clin Biochem 1999; 32: 477-80.

14. Bais R. The effect of sample hemolysis on cardiac troponin I and T Assays. Clin Chem 2010; 56: 1357-9

15. Jesse RL, Babb JD, Christenson RH, et al. Expert consensus document ACCF 2012 Expert Consensus Document on Practical Clinical Considerations in the Interpretation of Troponin Elevations. 2012; 60(23).

16. Jaffe AS, Wu AH. Troponin release-reversible or irreversible injury? Clin Chem 2012; 58: 148-50.

17. Kahn NA, Hemmelgam BR, Tonelli M, et al. Prognostic Value of Troponin T and I Among Asymptomatic Patients with End-Stage Renal Disease a Meta-Analysis. Circulation. 2005; 112: 3088

18. Stacy SR, Suarez-Cuervo C, Berger Z, Wilson LM, Yeh HC, Bass EB, Michos ED. Role of Troponin in Patients With Chronic Kidney Disease and Suspected Acute Coronary Syndrome. Ann Intern Med. 2014; 161: 502-512.

19. Abbas NA, John RI, Webb MC, et al. Cardiac troponins and renal function in nondialysis patients with chronic kidney disease

20. Wang AY, Lam CW, Wang M, et al. Prognostic value of cardiac troponin T is independent of inflammation, residual renal function, and cardiac hypertrophy and dysfunction in peritoneal dyalisis patients. Clin Chem 2007; 53: 882.

21. Anavekar NS, McMurray JJ, Velázquez EJ, Solomon SD, Kober L, Rouleau JL, et al. Relation between renal disfunction and cardiovascular outcome after myocardial infarction. N Engl J Med 2004; 351:1258-1295.

22. Muntner P, He J, Hamm L, Loria C, Whelton PK, et al. J Renal insufficiency and subsequent death resulting from cardiovascular disease in the United States. J Am Soc Nephrol 2002; 13: 745.

23. Donnino MW, Karriem-Norwood V, Rivers EP, et al. Prevalence of Elevated Troponin I in End-stage Renal Disease Patients Receiving Hemodialysis. Acad Emerg Med: 11; 979. 